\title{
Comprehensive appraisal of cardiac motion artefact in optical coherence tomography
}

\author{
Miao Chu ${ }^{1,2 *(0)}$, Carlos Cortés ${ }^{3,4 *}$ (), Lili Liu ${ }^{1,5}$, Miguel Ángel Martínez-Hervás-Alonso², \\ Bernd Reisbeck ${ }^{2,6}$, Ruiyan $Z_{\text {hang }}^{5}$, Shengxian Tu ${ }^{1}$, Juan Luis Gutiérrez-Chico ${ }^{2,3,5,7}$ (อ) \\ ${ }^{1}$ Med-X Research Institute, School of Biomedical Engineering, \\ Shanghai Jiao Tong University, Shanghai, China \\ ${ }^{2}$ Cardiology Department, Campo de Gibraltar Health Trust, Algeciras, Spain \\ ${ }^{3}$ Klinikum Frankfurt (Oder), Frankfurt (Oder), Germany \\ ${ }^{4}$ Miguel Servet University Hospital, Zaragoza, Spain \\ ${ }^{5}$ Department of Cardiology, Ruijin Hospital, Shanghai Jiao Tong \\ University School of Medicine, Shanghai, China \\ ${ }^{6}$ Cardio-Care Heart Center, Marbella, Spain \\ ${ }^{7}$ DRK Klinikum Westend, Berlin, Germany
}

\begin{abstract}
Background: The relation between cardiac motion artefact (CMA) in optical coherence tomography $(O C T)$ and the phases of cardiac cycle is unclear.

Methods: Optical coherence tomography pullbacks containing metallic stents were co-registered with angiography and retrospectively analyzed. The beginning of three phases, namely ejection, rapid-inflow and diastasis, was identified in angiography. Rotation, shortening, elongation and repetition were qualitatively labelled as CMA artefacts. Platforms with coaxial longitudinal connectors (ML8 and Magmaris) entered a quantitative sub-study, consisting of measuring the length of their connector at the beginning of each phase.

Results: A total of 261 stents (127 patients) were analyzed, including 105 stents for quantitative sub-study. CMA was detected in 61 (23.4\%) stents: rotation in 6 (2.3\%), shortening in 50 (19.2\%), elongation in 51 (19.5\%) and repetition in 12 (4.6\%). Shortening was always observed during ejection phase, while elongation and repetition were always observed during rapid-inflow. Rotation occurred in both ejection and rapid-inflow phases, while no artefact was reported during diastasis. Longitudinal connectors measured in early ejection phase and in early rapid-inflow phase were shorter and longer, respectively, than those measured in diastasis, irrespective of the presence of CMA in the qualitative assessment.

Conclusions: Cardiac motion artefact is prevalent in OCT studies, but shortening and elongation of vascular structures occur during early ejection and during early rapid-inflow, respectively, to a greater or lesser extent in all cases. Diastasis is free of CMA and hence the period in which longitudinal measurements can be more accurately quantified. (Cardiol J)
\end{abstract}

Key words: tomography, optical coherence, artefact, percutaneous coronary intervention, coronary heart disease, stents

Address for correspondence: Prof. Juan Luis Gutiérrez-Chico, MD, PhD, FESC, FACC, Head of Interventional Cardiology, Ruijin Hospital, 197 Ruijin 2nd Road, Huangpu District, 200025 - Shanghai, China, tel: +34 615319370 , e-mail: juanluis.gutierrezchico@ictra.es

Received: 2.07.2021 Accepted: 24.10.2021 Early publication date: 26.10.2021

*These authors have equally contributed.

This article is available in open access under Creative Common Attribution-Non-Commercial-No Derivatives 4.0 International (CC BY-NC-ND 4.0) license, allowing to download articles and share them with others as long as they credit the authors and the publisher, but without permission to change them in any way or use them commercially. 


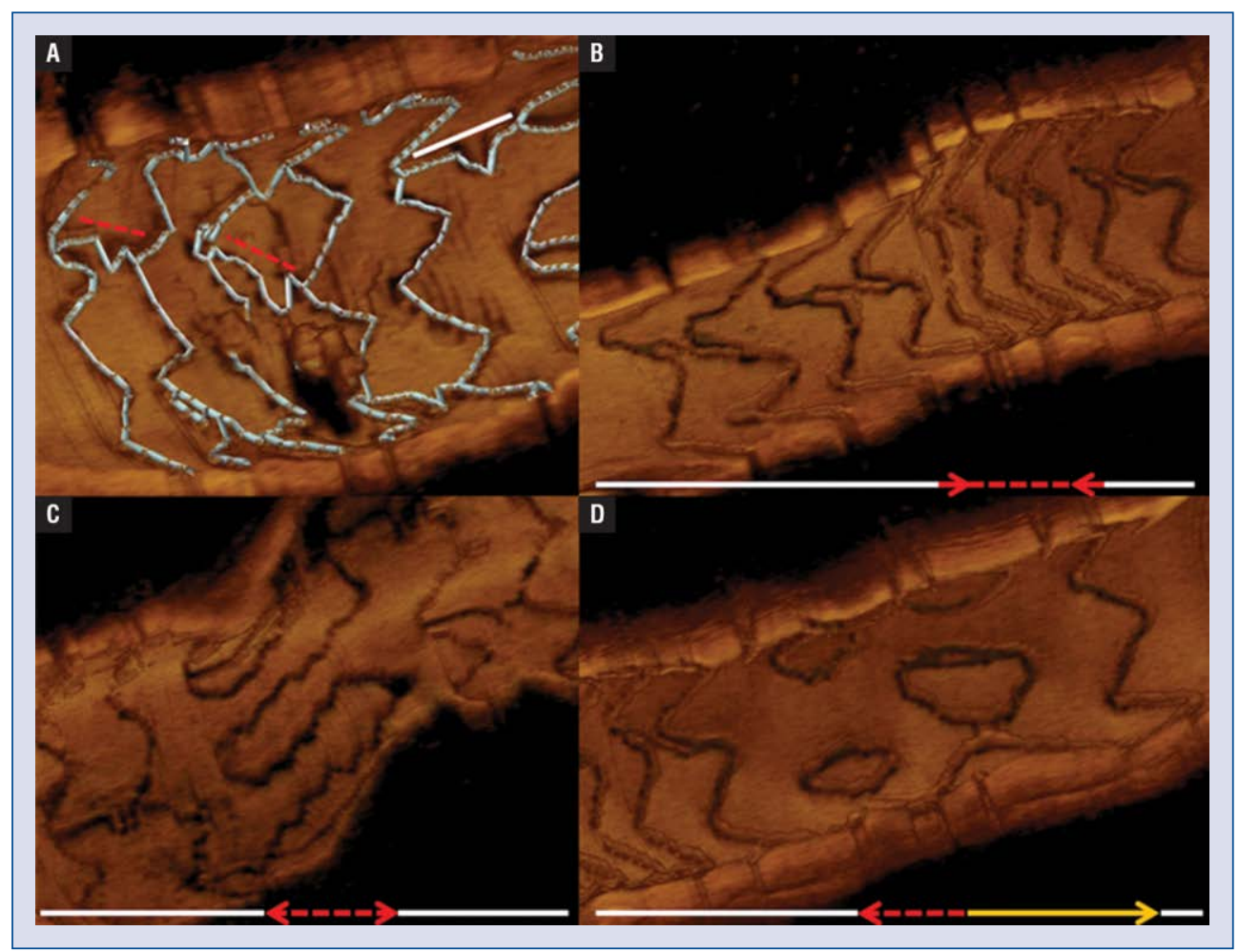

Figure 1. Four different subtypes of cardiac motion artefact are considered in the current study. Rotation (A), defined as twisting of the stent structure around its longitudinal axis in the longitudinal view or in the three-dimensional (3D) reconstruction. Notice the longitudinal connector of the ML8/Vision/Xience platform (white line), changing direction and misaligning with the vessel longitudinal axis (red dotted lines). Shortening (B) and elongation (C), defined as any modular element of the stent appearing relatively shorter (B, dotted arrows) or longer ( $\mathbf{C}$, dotted arrows), respectively, than the average length of identical modular elements in the longitudinal view or in the 3D reconstruction (B, $\mathbf{C}$, white lines). Repetition (D), defined as substantial distortion of the stent structure due to repeated scanning of the same elements backwards (red dotted arrow) and forward again (yellow arrow).

\section{Introduction}

Cardiac motion artefact (CMA) has been described in three-dimensional (3D) optical coherence tomography (OCT) as the longitudinal distortion of coronary structures due to systo-diastolic movements over the cardiac cycle [1,2]. Albeit the pullback speed in OCT is even and constant, the relative speed of the optical catheter with respect to the coronary artery changes over the cardiac cycle, thus causing longitudinal distortion to the 3D-OCT reconstruction [1-3]. Previous pioneer studies on CMA have described three subtypes of CMA artefact: rotation, elongation and repetition, linking them to the early ejection phase of systole, because the contraction of the vessel moved the vascular structures in the same direction as the catheter pullback, thus reducing the relative pullback speed and subsequently elongating or even duplicating some imaged elements [2]. Nonethe- less, these pioneer descriptions of CMA were performed on systems without co-registration, neither with electrocardiogram (ECG) nor with angiography. Therefore, this plausible hypothesis has never been verified hitherto.

Co-registration of OCT with coronary angiography is currently widely available, thus enabling a detailed analysis of the longitudinal distortion caused by the different phases of the cardiac cycle. Revisiting this phenomenon on the light of co-registered OCT studies, especially involving imaging of intracoronary stents, might challenge previous conceptions about CMA. Based on the observation of co-registered 3D-OCT studies, we propose four different subtypes of CMA artefact, namely rotation, shortening, elongation and repetition (Fig. 1). The current study is aimed to explore the association between the different subtypes of CMA artefact to each specific phase of the cardiac cycle (Fig. 2 and Central illustration). 


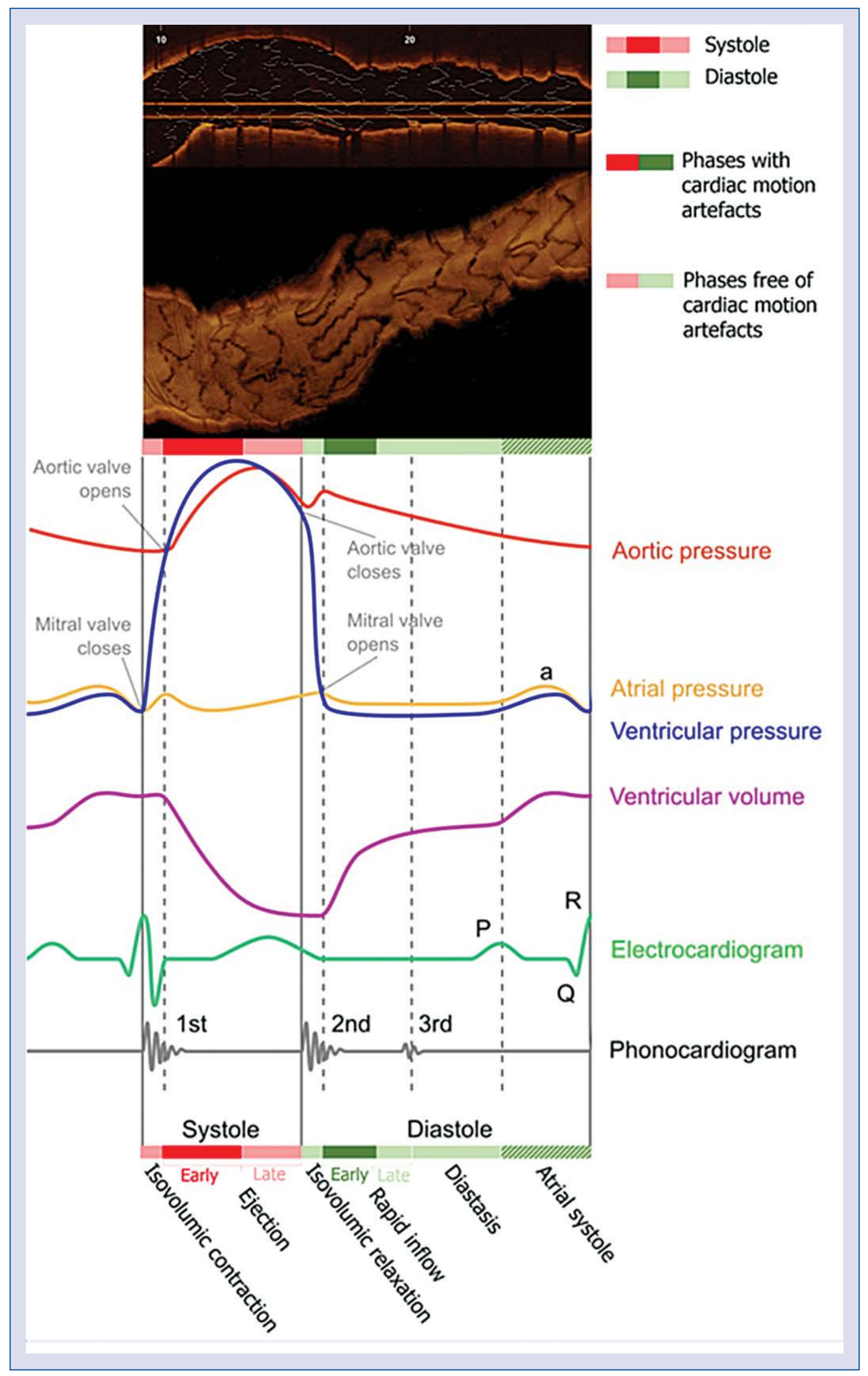

Figure 2. Correspondence of cardiac motion artefact with the phases of the cardiac cycle. All subtypes of cardiac motion artefact were detected on the early ejection-phase of systole (shortening, rotation) or in the early rapid-inflow phase of diastole (elongation, repetition, rotation). Elongation and repetition have been observed in some cases during the atrial contraction. The rest of the phases in the cardiac cycle are free of cardiac motion artefact. 


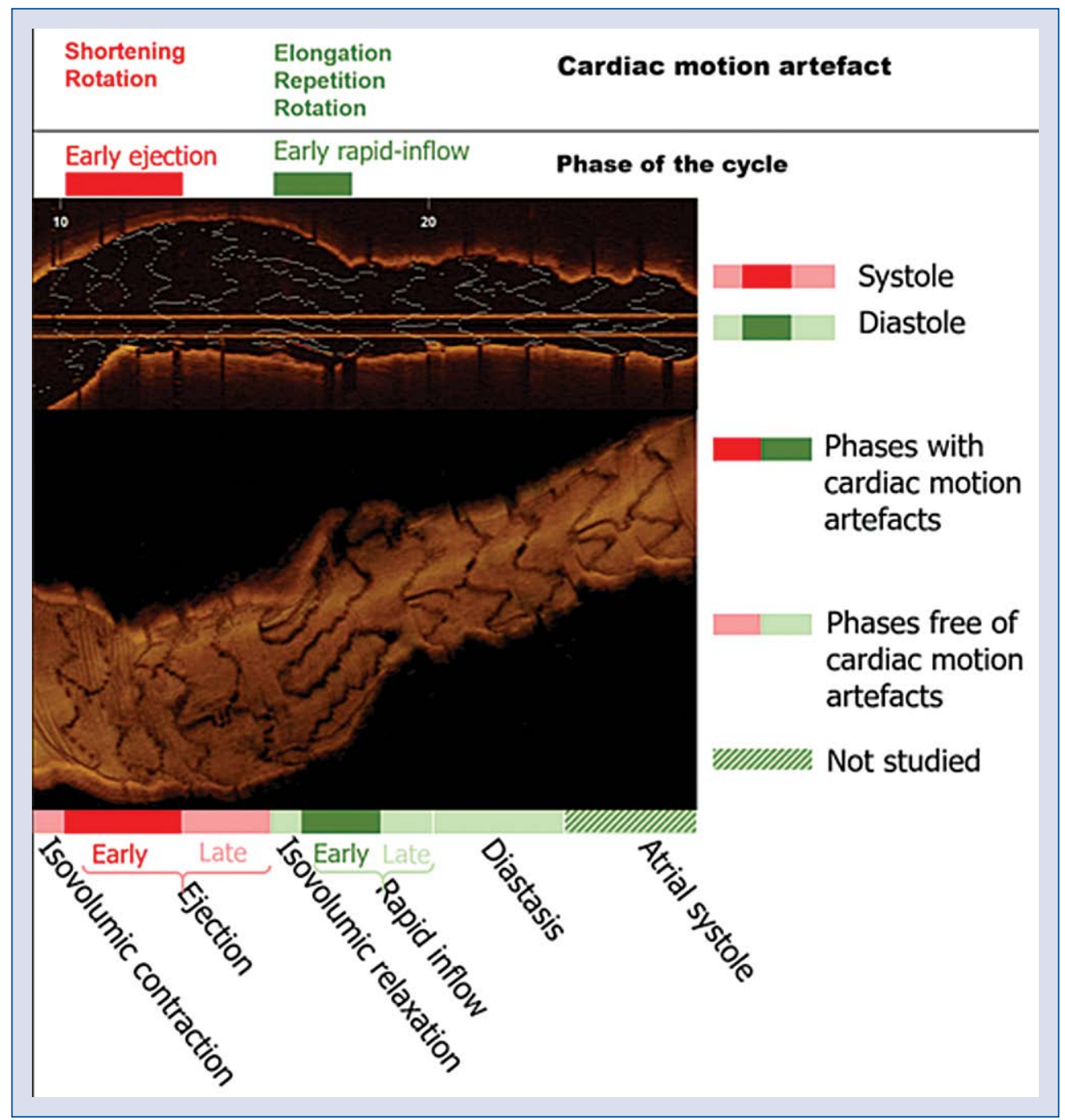

Central illustration. Zoomed correspondence of cardiac motion artefact with the phases of the cardiac cycle. All subtypes of cardiac motion artefact were detected on the early ejection-phase of systole (shortening, rotation) or in the early rapid-inflow phase of diastole (elongation, repetition, rotation). Elongation and repetition have been observed in some cases during the atrial contraction. The rest of the phases in the cardiac cycle are free of cardiac motion artefact.

\section{Methods}

Consecutive patients undergoing OCT of a coronary artery previously treated with implantation of a metallic stent, either durable or bioresorbable, in any of the three participating centers (Klinikum Frankfurt Oder, Germany; DRK Klinikum Westend, Berlin, Germany and Campo de Gibraltar Health Trust, Algeciras, Spain) between 01-03-2016 and 01-08-2019 were retrospectively included into the study. Exclusion criteria were: 1) previous treatment of the target vessel with non-metallic bioresorbable scaffolds (BRS) alone; 2) overlapping stents or multiple stent layers leaving
$<5$ mm monolayer segment; 3) poor OCT quality for the analysis due to non-uniform rotational distortion (NURD), suboptimal vessel flushing, incomplete purge of the optic catheter or other artefacts; 4) severe stent distortion due to longitudinal stress or collapse of the lumen, leaving $<5 \mathrm{~mm}$ of stent structurally preserved and suitable for analysis; 5) missing co-registration with angiography. All OCT studies were acquired with a Dragonfly ${ }^{\mathrm{TM}}$ catheter and an ILUMIEN OPTIS system (Abbott, St. Paul, Minnesota, USA), with a rotation speed of $180 \mathrm{~Hz}$ and a pullback speed of $18 \mathrm{~mm} / \mathrm{s}$ or $36 \mathrm{~mm} / \mathrm{s}$, resulting in longitudinal resolutions of 0.1 and $0.2 \mathrm{~mm}$, respectively, calculated as pull- 


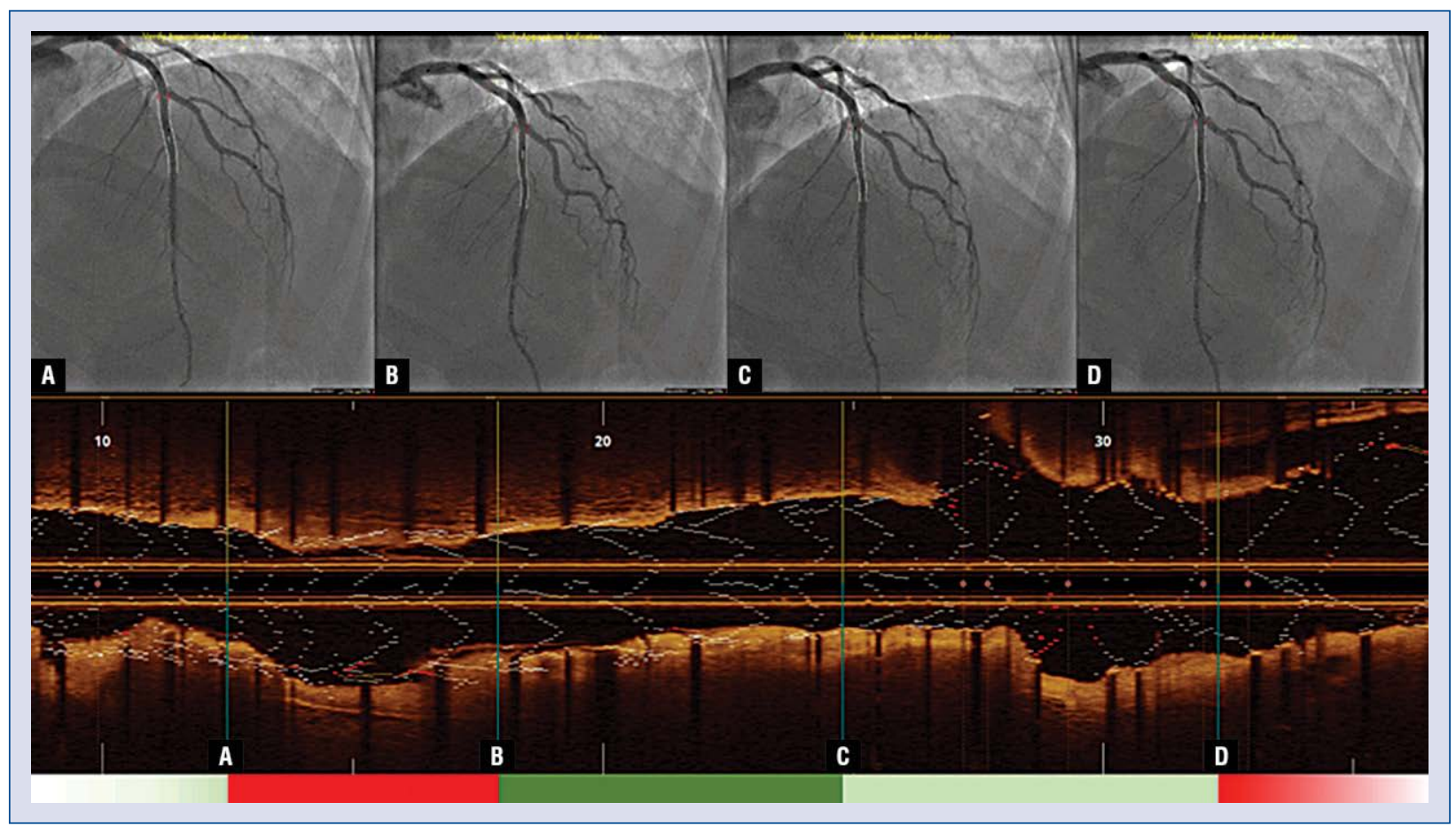

Figure 3. Approximation to the cardiac cycle phases using angiographic co-registration. Beginning of the ejection phase (A, D): First frame showing contraction of the coronary vessels. Beginning of the rapid-inflow phase (B): First frame showing distension of the coronary vessels after systole. Beginning of diastasis (C): First frame in which the coronary arteries have reached their maximal expansion during diastole, before the atrial contraction. These three landmarks divide the cardiac cycle into three periods: 1) systolic period ( $A-B$, red stripe), encompassing the ejection phase + isovolumic relaxation phase; 2 ) rapid-inflow period ( $\mathrm{B}-\mathrm{C}$, dark green stripe), corresponding to the rapid-inflow phase, and 3) diastasis period (C-D, light green stripe), encompassing diastasis, atrial contraction and isovolumic contraction.

back speed $(\mathrm{mm} / \mathrm{s})$ divided by rotation speed $(\mathrm{Hz}$ or $\mathrm{s}^{-1}$ or cross-sections/s). All cases were acquired with non-occlusive technique [4] and automatic contrast injection, calculating the contrast volume by a validated formula to optimise the image quality with a minimal amount of dye [5]. Clinical information about patients and procedures was retrospectively collected from clinical recordings at each center.

The study complied with the principles of good clinical practice and with the Declaration of Helsinki for investigation in human beings. The study protocol was approved by the corresponding institutional review boards.

\section{Phases of the cardiac cycle using angiographic co-registration}

Since co-registration with ECG was not available, the different phases of the cardiac cycle had to be approximated by means of co-registration with coronary angiography, similarly to previous studies
[6]. Three different moments of the cardiac cycle were identified in angiography by analysing the movement of the coronary vessels: beginning of the ejection phase, beginning of the rapid-inflow phase and beginning of diastasis (Fig. 2). The beginning of the ejection phase was identified as the first angiographic frame showing longitudinal contraction of the coronary vessels. The beginning of the rapidinflow phase was identified as the first angiographic frame showing distension of the coronary vessels after systole. Finally, the beginning of diastasis was identified as the first angiographic frame in which the coronary arteries reached their maximal expansion during diastole, before the atrial contraction.

These three landmarks divide the cardiac cycle into three periods: 1) systolic period, encompassing the ejection phase, but also the isovolumic relaxation phase of diastole, 2) the rapid-inflow period, and 3) the diastasis period, encompassing diastasis, but also atrial contraction and isovolumic contraction phase of systole (Fig. 3). 


\section{Qualitative assessment of cardiac} motion artefact

Optical coherence tomography raw data were evaluated by two independent analysts, blinded to each other results, using an Ilumien Optis workstation (Abbott, St. Paul, Minnesota, USA) equipped with longitudinal view, automatic strut detection and 3D-OCT. Both analysts knew in full detail the structural design of the stent platforms imaged in the study, as previously described [7]. After identification of the stented segment and the corresponding analysable monolayer (in case of overlapping), selecting the most appropriate cropping plane, the analysts qualitatively assessed the presence of CMA on the longitudinal view or in 3D-OCT, as any of the following four types of distortion: rotation, shortening, elongation and repetition (Fig. 1). The analysis on the longitudinal view required the use of automatic strut detection, while it was optional on 3D-OCT [7].

Rotation was defined as twisting of the stent structure around its longitudinal axis in the longitudinal view or in the 3D reconstruction. Shortening and elongation were defined as any modular element of the stent appearing relatively shorter or longer, respectively, than the average length of identical modular elements in the longitudinal view or in the 3D reconstruction (Fig. 1). Repetition was defined as substantial distortion of the stent structure due to repeated scanning of the same elements backwards and forward again (Fig. 4).

The analysts annotated the period of the cycle, as defined by angiography, in which CMA was detected. Due to the methodological difficulties to identify atrial contraction using angiography, this phase of the cycle was excluded from the analysis, by disregarding any artefact observed in the second half of the diastasis period defined by angiography.

\section{Quantitative assessment of cardiac motion artefact}

Quantification was restricted to only two different stent platforms: ML8/Vision/Xience and Magmaris platforms [7], because their design contains longitudinal connectors, aligned in parallel to the longitudinal axis of the stent, thus enabling more accurate length measurements than other stent designs. The analysts measured the length of the first longitudinal connector imaged in its full length in the systolic period, the rapid-inflow period and the diastasis period, defined by the co-registered angiography, using the longitudinal view (Fig. 5), thus corresponding to the early ejec-

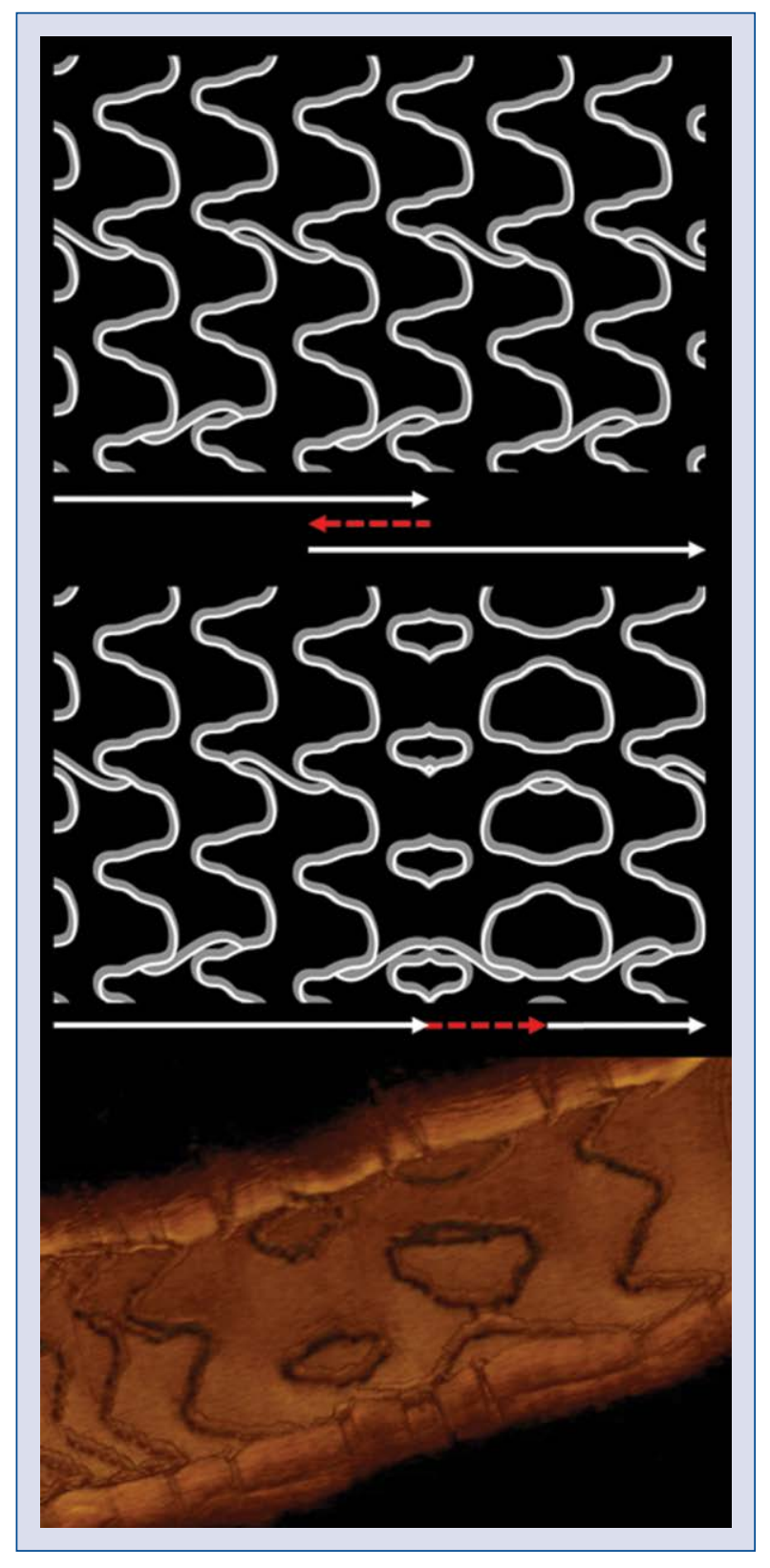

Figure 4. Mechanism of the repetition artefact. The upper panel show the structure of a Magmaris scaffold. During the optical coherence tomography (OCT) scanning (white arrows), at the early rapid-inflow phase of diastole, the relative pullback speed of the optical source in relation to the tissue can be reversed and take negative values, thus resulting in backward scanning of previously imaged elements (red dotted arrow), that are re-imaged forward again at subsequent phases of the cycle, as soon as the relative pullback speed becomes positive again. As a result, some segments are scanned three times (forth-back-forth). Since OCT displays the scanning as a continuous image in the same direction (mid-panel), every change in the direction of scanning produces a specular pattern of the previously imaged elements, resulting in the typical kaleidoscopic images of the repetition artefact (lower panel). 


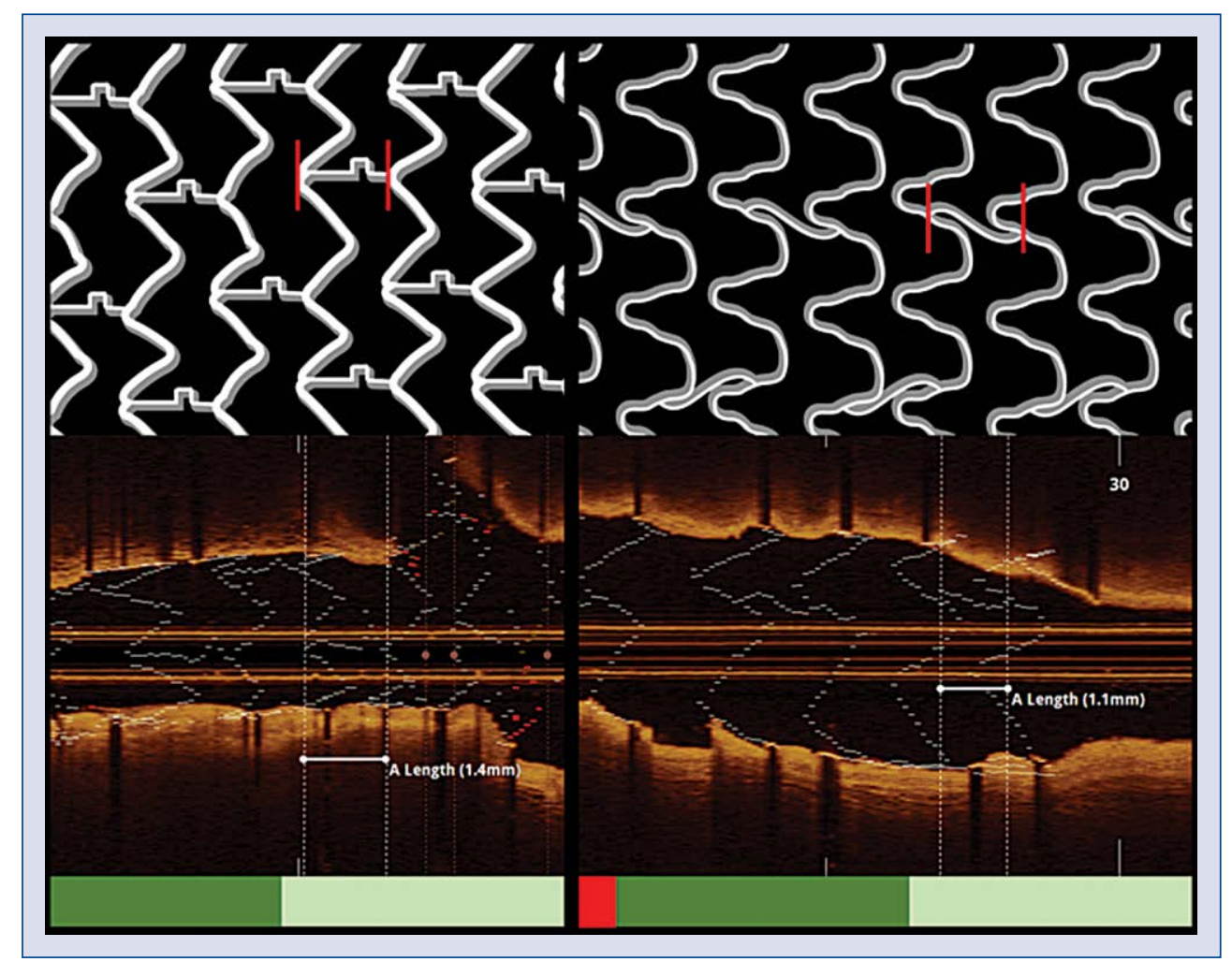

Figure 5. Quantitative measurements. The longitudinal connector of the ML8/Vision/Xience platform (left) and of the Magmaris platform (right) was measured in the longitudinal view. The example shows measurement of the first connector in diastasis period, defined by angiography. It is important to measure at initial parts of the period to avoid atrial and isovolumic contraction, occuring at the final part of the angiographic period.

tion phase, the early rapid-inflow phase and the diastasis phase of the cardiac cycle, respectively. Stents with repetition artefact, with suboptimal longitudinal reconstruction of the stent or with insufficient length as to show a measurable longitudinal connector in at least two different phases of the cycle were excluded from the quantitative analysis.

\section{Statistical analysis}

Descriptive statistics of continuous variables were reported as mean \pm standard deviation (SD) if they followed a Gaussian distribution or as median (quartiles) if differently distributed, while those of categorical variables were presented as counts (percentages). The length of the longitudinal connectors at different cardiac phases were compared with Student's t-test for paired measurements; dichotomous and categorical variables were compared with Pearson's $\chi^{2}$ or with Fisher's exact test if the expected count was $<5$ in any cell. Subgroup analysis stratified by pullback speed was performed. Interobserver reproducibility was reported as kappa coefficient for the qualitative assessment and as intraclass correlation coefficient for the absolute measurement (ICCa) for the quantitative assessment.

All analysis was performed with IBM SPSS 24.0 software package (SPSS Inc., Chicago, Illinois).

\section{Results}

A total of 193 patients underwent OCT studies in the enrolling centres during the study period. Sixty-six patients (68 studies) were excluded: 40 because no stent was imaged in the OCT study (58.8\%), $22(32.4 \%)$ because only non-metallic BRS were implanted in the intervention, $3(4.4 \%)$ due to incomplete blood clearance, $2(2.9 \%)$ due to severe stent distortion and $1(1.5 \%)$ due to NURD. During the analysis 19 stents were excluded due to multilayer (6), overlap with $<5 \mathrm{~mm}$ of monolayer (5), co-registration missing (3), suboptimal vessel flushing (3), incomplete purge of the optic catheter (1) or NURD (1). A total of 127 patients, 132 procedures, 152 lesions, 166 pullbacks and 261 stents were finally analysed (Fig. 6). 


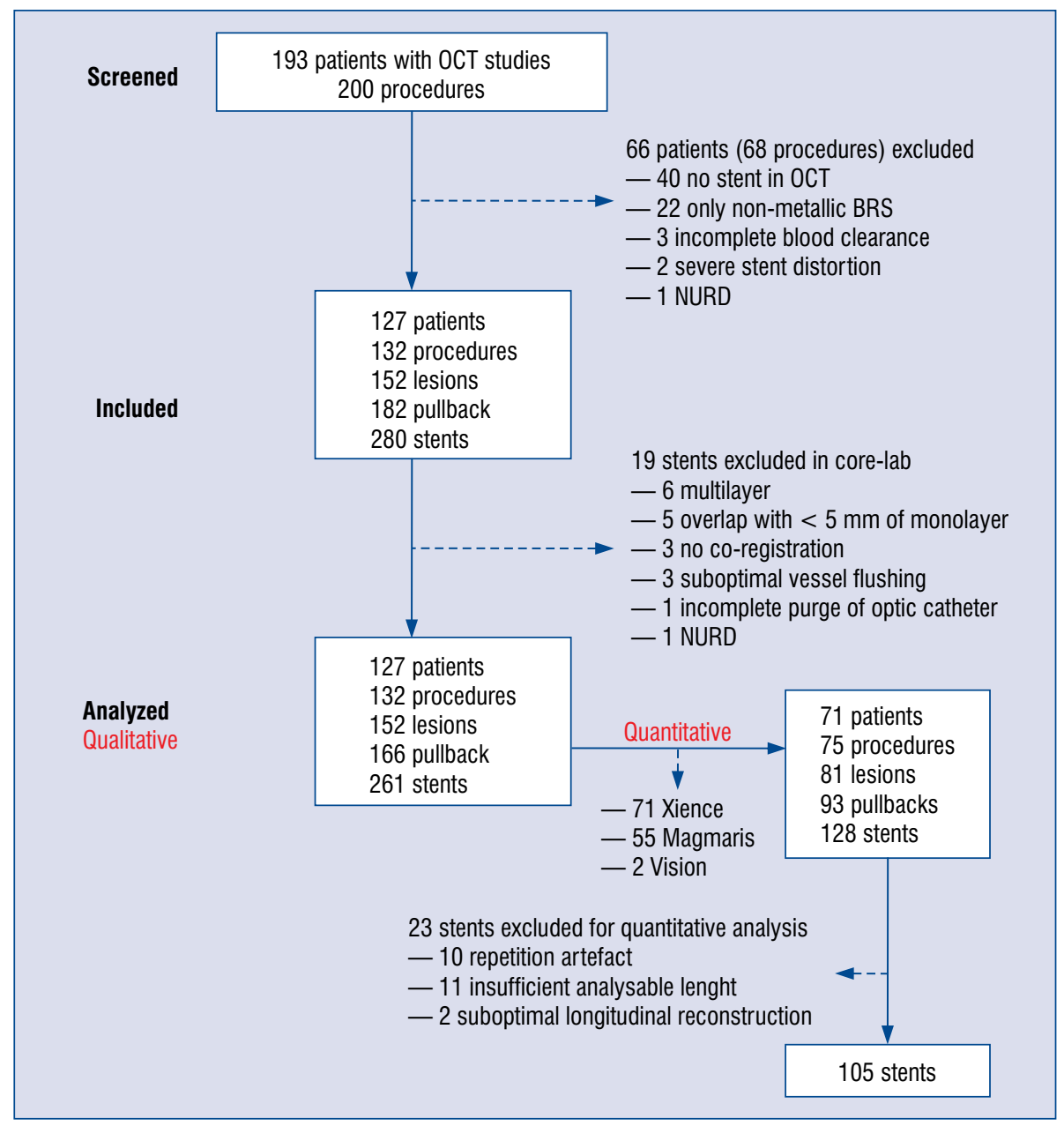

Figure 6. Study flow-chart; BRS — bioresorbable scaffolds; OCT — optical coherence tomography; NURD — nonuniform rotational distortion.

\section{Descriptive statistics of the sample}

Tables 1 and 2 present the descriptive statistics of the sample. Most stents were implanted in the left anterior descending $(41.0 \%)$ or the right coronary artery (30.3\%). Different types of stents were imaged and analyzed in this study (Table 2), but the most common platforms were ML8/Vision/ /Xience (71 Xience, 27.2\%; 2 Vision, 0.8\%; Abbott Vascular, Santa Clara, CA) and Magmaris (55 Magmaris, 21.1\%; Biotronik AG, Bülach, CH). Ninety-nine $(37.9 \%)$ stents were implanted more than 3 months prior to the OCT study and 62 (23.9\%) presented in-stent restenosis as anatomic substrate for the clinical symptoms. Most studies (87.7\%) were acquired at a pullback speed of $18 \mathrm{~mm} / \mathrm{s}$.

\section{Qualitative assessment}

Cardiac motion artefact was identified in 61 (23.4\%) stents, corresponding to $6(2.3 \%)$ cases of rotation, $50(19.2 \%)$ cases of shortening, 51 (19.5\%) cases of elongation and $12(4.6 \%)$ cases of repetition (Table 3). The incidence of CMA was significantly higher at low pullback speed $(18 \mathrm{~mm} / \mathrm{s}: 25.3 \%)$ than at high pullback speed $(36 \mathrm{~mm} / \mathrm{s}: 9.4 \%)$. In the subgroup analysis, both shortening and elongation occurred more frequently at low pullback speed. Repetition was only observed at $18 \mathrm{~mm} / \mathrm{s}$, with low incidence $(5.2 \%)$. Rotation was observed at both pullback speeds, with low incidence and without significant differences.

Shortening was only detected during the systolic period, while elongation and repetition were only detected during the rapid-inflow period. Rotation, however, was detected during the systolic period ( 3 cases, $50 \%$ ), the rapid-inflow period ( 1 case, $16.7 \%$ ) or in both periods ( 2 cases, $33.3 \%$ ). No artefact was reported during the diastasis period (Table 3).

Reproducibility was excellent for repetition (kappa 1.000; 95\% confidence interval [CI] 0.999- 
Table 1. Descriptive statistics of patients, intervention and lesions.

\begin{tabular}{|c|c|}
\hline Patient level & $N=127$ \\
\hline Male, n (\%) & $99(78.0)$ \\
\hline Age [years] & $67.2(58.5-75.3)$ \\
\hline Body mass index $\left[\mathrm{kg} / \mathrm{m}^{2}\right]$ (SD) & $28.7(4.7)$ \\
\hline \multicolumn{2}{|l|}{ Cardiovascular risk factors: } \\
\hline Hypertension & $103(81.1)$ \\
\hline Hypercholesterolemia & $64(50.4)$ \\
\hline \multicolumn{2}{|l|}{ Diabetes mellitus: } \\
\hline Type 2 on OAD & $38(29.9)$ \\
\hline Type 2 insulin-requiring & $11(8.7)$ \\
\hline \multicolumn{2}{|l|}{ Smoking: } \\
\hline Previous smoker & $26(20.5)$ \\
\hline Current smoker & $28(22.0)$ \\
\hline Family history of CHD & $6(4.7)$ \\
\hline Previous MI & $53(41.7)$ \\
\hline \multicolumn{2}{|l|}{ Previous revascularization: } \\
\hline $\mathrm{PCl}$ & $80(63.0)$ \\
\hline CABG & $9(7.1)$ \\
\hline GFR (Cockroft-Gault) [mL/min] & $87.7(48.5)$ \\
\hline Serum hemoglobin $[\mathrm{g} / \mathrm{dL}]$ & $13.5(1.7)$ \\
\hline LVEF [\%] & $60(12)$ \\
\hline Procedural variables & $N=132$ \\
\hline Syntax score & $13.7(8.6)$ \\
\hline Contrast volume [mL] & $232(106)$ \\
\hline Fluoroscopy time [min] & $20.8(15.8)$ \\
\hline \multicolumn{2}{|l|}{ Clinical indication } \\
\hline Stable coronary disease & $98(74.2)$ \\
\hline Unstable angina & $15(11.4)$ \\
\hline NSTEMI & $18(13.6)$ \\
\hline STEMI & $1(0.8)$ \\
\hline Lesions & $N=152$ \\
\hline \multicolumn{2}{|l|}{ Calcification: } \\
\hline None to little & $130(85.5)$ \\
\hline Moderate to severe & $22(14.5)$ \\
\hline Diameter stenosis [\%] & $72.2(15.9)$ \\
\hline
\end{tabular}

Data presented as counts (percent), mean (standard deviation) or median $\left(\mathrm{P}_{25}-\mathrm{P}_{75}\right)$. CABG - coronary artery bypass grafting; $\mathrm{CHD}$ - coronary heart disease; GFR - glomerular filtration rate; LVEF - left ventricular ejection fraction; MI - myocardial infarction; NSTEMI - non-ST-segment elevation myocardial infarction; $\mathrm{OAD}$ - oral antidiabetics; $\mathrm{PCl}$ - percutaneous coronary intervention; STEMI - ST-segment elevation myocardial infarction

$-1.000)$ and moderate for other artefacts: shortening (kappa 0.469; 95\% CI 0.347-0.591), elongation (kappa $0.534 ; 95 \%$ CI $0.420-0.648$ ), rotation (kappa 0.606; 95\% CI 0.288-0.924).
Table 2. Descriptive statistics of the analyzed stents.

\begin{tabular}{|c|c|}
\hline Stents analyzed & $N=261$ \\
\hline \multicolumn{2}{|l|}{ Coronary artery: } \\
\hline Left main & $10(3.8)$ \\
\hline Left anterior descending & $107(41.0)$ \\
\hline Diagonal & $12(4.6)$ \\
\hline Circumflex & $42(16.1)$ \\
\hline Obtuse marginal & $9(3.4)$ \\
\hline Right coronary artery & 79 (30.3) \\
\hline Posterolateral & $2(0.8)$ \\
\hline \multicolumn{2}{|l|}{ Type of stent implanted: } \\
\hline Xience & $71(27.2)$ \\
\hline Magmaris & $55(21.1)$ \\
\hline Biofreedom & $23(8.8)$ \\
\hline Resolute Integrity & $19(7.3)$ \\
\hline Orsiro & $17(6.5)$ \\
\hline Coroflex & $14(5.4)$ \\
\hline Promus Element & $12(4.6)$ \\
\hline Bioss-Lim C & $10(3.8)$ \\
\hline ML Rx Pixel & $9(3.4)$ \\
\hline Resolute Onyx & $7(2.7)$ \\
\hline Driver & $6(2.3)$ \\
\hline Taxus Liberté & $4(1.5)$ \\
\hline Biomatrix & $3(1.1)$ \\
\hline ML Zeta & $2(0.8)$ \\
\hline Vision & $2(0.8)$ \\
\hline Biodivysio & $2(0.8)$ \\
\hline Alex Plus & $2(0.8)$ \\
\hline Cypher & $1(0.4)$ \\
\hline Taxus Express & $1(0.4)$ \\
\hline Costar & $1(0.4)$ \\
\hline \multicolumn{2}{|l|}{ Timing of implant: } \\
\hline Recently implanted (< 3 months) & $162(62.1)$ \\
\hline Late implanted ( $\geq 3$ months) & 99 (37.9) \\
\hline Immediately post-implant & $141(54.0)$ \\
\hline $\begin{array}{l}\text { Time from stent implantation } \\
\text { [months] }^{*}\end{array}$ & $\begin{array}{c}25.3 \\
(7.4-78.0)\end{array}$ \\
\hline In-stent restenosis & $62(23.8)$ \\
\hline \multicolumn{2}{|l|}{ Mehran's type**: } \\
\hline la & $1(1.6)$ \\
\hline $\mathrm{lb}$ & $3(4.8)$ \\
\hline Ic & $11(17.7)$ \\
\hline Id & $0(0.0)$ \\
\hline II & $27(43.6)$ \\
\hline III & $18(29.0)$ \\
\hline IV & $2(3.2)$ \\
\hline Overlap & $119(45.6)$ \\
\hline \multicolumn{2}{|l|}{ Pullback speed: } \\
\hline $18 \mathrm{~mm} / \mathrm{s}$ & $229(87.7)$ \\
\hline $36 \mathrm{~mm} / \mathrm{s}$ & $32(12.3)$ \\
\hline
\end{tabular}

Data presented as counts (percent) or median $\left(\mathrm{P}_{25}-\mathrm{P}_{75}\right)$. ${ }^{*}$ For the group of late implanted stents; **For the subgroup with in-stent restenosis. 
Table 3. Cardiac motion artefact, stratified by pullback (PB) speed and relation with the period of cardiac cycle (as defined by co-registration with angiography).

\begin{tabular}{|c|c|c|c|c|c|c|c|}
\hline & \multirow{2}{*}{$\begin{array}{c}\text { Total } \\
(n=261)\end{array}$} & \multicolumn{2}{|c|}{ PB speed } & \multirow[t]{2}{*}{$\mathbf{P}$} & \multicolumn{3}{|c|}{ Period } \\
\hline & & $\begin{array}{l}18 \mathrm{~mm} / \mathrm{s} \\
(\mathrm{n}=229)\end{array}$ & $\begin{array}{l}36 \mathrm{~mm} / \mathrm{s} \\
(\mathrm{n}=32)\end{array}$ & & Systolic & Rapid-inflow & Diastasis \\
\hline Cardiac motion artefact: & $61(23.4)$ & $58(25.3)$ & $3(9.4)$ & 0.046 & $5(1.9)$ & & \\
\hline Rotation & $6(2.3)$ & $4(1.7)$ & $2(6.3)$ & 0.160 & $50(19.2)$ & $3(1.1)$ & $0(0.0)$ \\
\hline Shortening & $50(19.2)$ & $50(21.8)$ & $0(0.0)$ & 0.003 & $0(0.0)$ & $0(0.0)$ & $0(0.0)$ \\
\hline Elongation & $51(19.5)$ & $49(21.4)$ & $2(6.3)$ & 0.043 & $0(0.0)$ & $51(19.5)$ & $0(0.0)$ \\
\hline Repetition & $12(4.6)$ & $12(5.2)$ & $0(0.0)$ & 0.371 & $0(0.0)$ & $12(4.6)$ & $0(0.0)$ \\
\hline
\end{tabular}

Data presented as count (percent).

Table 4. Quantitative analysis of cardiac motion artefact (CMA), stratified by stent platform. Analysis in the whole sample (upper rows) and in the subgroup in which no CMA was detected in the qualitative analysis.

\begin{tabular}{|c|c|c|c|c|c|c|c|c|c|}
\hline & \multicolumn{7}{|c|}{ Length of longitudinal connector [mm] } & \multirow{3}{*}{$\begin{array}{l}\text { Ejection } \\
\text { vs. } \\
\text { diastasis } \\
\text { (p) }\end{array}$} & \multirow{3}{*}{$\begin{array}{l}\text { Rapid- } \\
\text { inflow } \\
\text { vs. diastasis } \\
\text { (p) }\end{array}$} \\
\hline & \multicolumn{2}{|c|}{$\begin{array}{c}\text { Early ejection } \\
\text { phase }\end{array}$} & \multicolumn{2}{|c|}{$\begin{array}{l}\text { Early rapid- } \\
\text {-inflow phase }\end{array}$} & \multicolumn{3}{|c|}{ Diastasis } & & \\
\hline & Mean & SD & Mean & SD & Mean & Mode & SD & & \\
\hline ML8/Vision/Xience $(n=57)$ & 1.13 & 0.20 & 1.89 & 0.41 & 1.43 & 1.40 & 0.06 & $<0.0001$ & $<0.0001$ \\
\hline Magmaris $(n=48)$ & 0.85 & 0.16 & 1.58 & 0.45 & 1.13 & 1.10 & 0.05 & $<0.0001$ & $<0.0001$ \\
\hline \multicolumn{10}{|l|}{ Subgroup no CMA } \\
\hline ML8/Vision/Xience $(n=36)$ & 1.21 & 0.12 & 1.68 & 0.21 & 1.42 & 1.40 & 0.05 & $<0.0001$ & $<0.0001$ \\
\hline Magmaris $(n=35)$ & 0.88 & 0.14 & 1.44 & 0.33 & 1.13 & 1.10 & 0.05 & $<0.0001$ & $<0.0001$ \\
\hline
\end{tabular}

Data presented as count (percent) of stents that were correctly identified. SD — standard deviation

\section{Quantitative analysis}

For the quantitative analysis, 73 stents with a ML8/Vision/Xience platform and 55 with a Magmaris platform were available. The analysts excluded 23 stents due to insufficient analysable length (11), repetition artefact (10) or suboptimal stent reconstruction in the longitudinal view (2), resulting in 105 devices finally analyzed in the quantitative sub-study: $57 \mathrm{ML} 8 /$ Vision/Xience and 48 Magmaris.

For both stent platforms, the longitudinal connector was significantly shorter during the early ejection phase than in diastasis and significantly longer during the early rapid-inflow-phase than in diastasis $(\mathrm{p}<0.0001$ for all comparisons; Table 4). The variability of the measurement was minimal during diastasis (SD $0.06 \mathrm{~mm}$ for ML8/ /Vision/Xience and $0.05 \mathrm{~mm}$ for Magmaris) and maximal during the early rapid-inflow-phase (SD $0.41 \mathrm{~mm}$ for ML8/Vision/Xience and $0.45 \mathrm{~mm}$ for Magmaris). In all the individual stents analyzed, the measurement of the longitudinal connector during the early ejection phase was $\leq$ than the measurement in diastasis, while the measurement during the early rapid-inflow phase was $\geq$ than in diastasis (Fig. 7), irrespective of the detection of CMA by the analyst (Table 4).

The reproducibility of quantitative measurements was very good: ICCa 0.898 (95\% CI 0.853-0.930) for the early ejection phase; ICCa 0.893 (95\% CI $0.845-0.926$ ) for the early rapidinflow phase and ICCa 0.911 (95\% CI 0.844-0.946) for diastasis.

\section{Discussion}

The main findings of the current study can be summarized as follows: 1) Four different types of distortion can be described as part of CMA, namely rotation, shortening, elongation and repetition, affecting both the 3D-OCT reconstruction and the longitudinal view (Fig. 1); 2) All of 


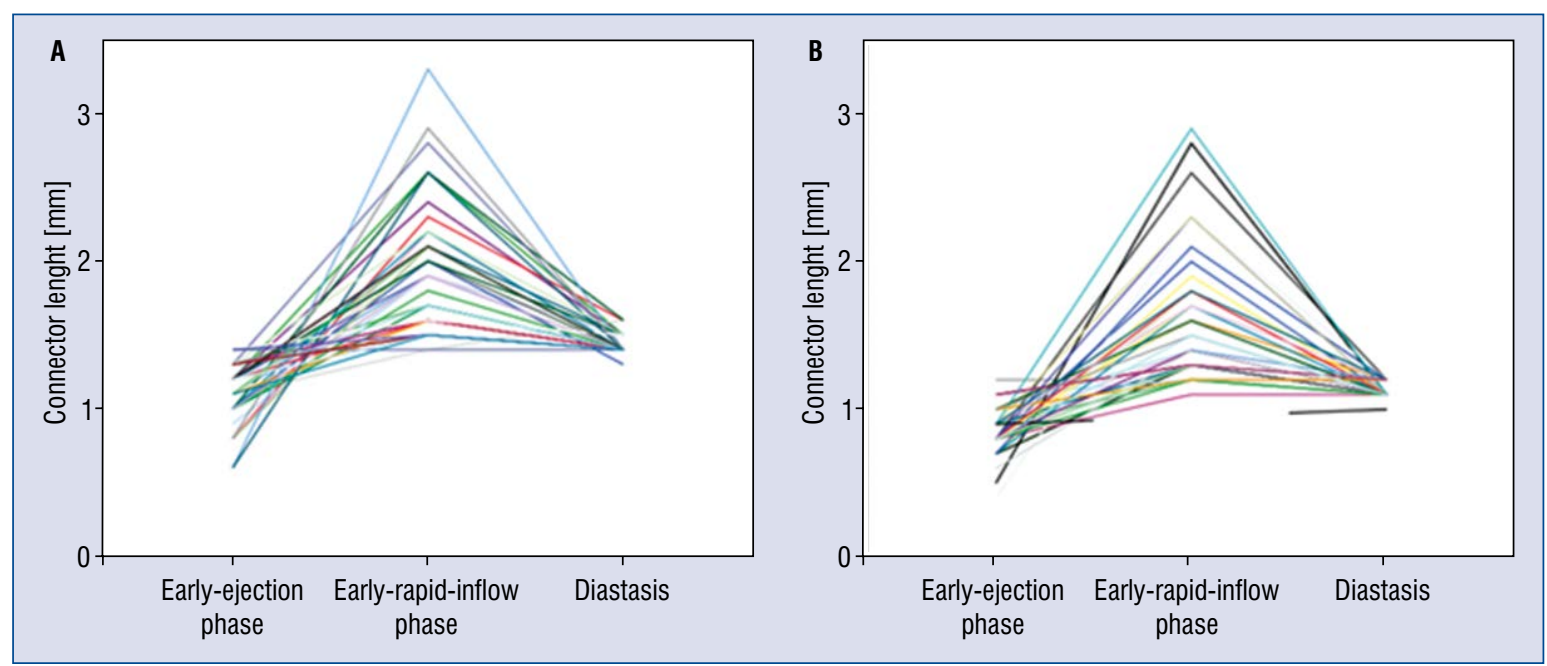

Figure 7. Per stent individual measurements of the longitudinal connector in different phases of the cardiac cycle. In practically all cases and for both platforms, the measurements were minimal at the early-ejection phase and maximal at the early rapid-inflow phase. The variability was maximal at the early rapid-inflow and minimal in diastasis; A. ML8/ Nision/Xience platform; B. Magmaris platform.

them, except rotation, occur more frequently at low pullback speed than at high pullback speed; 3) Shortening occurs at the early ejection phase of systole, while elongation and repetition occur at the early rapid-inflow phase of diastole; rotation, however, has been reported at both the ejection and the rapid-inflow phases (Central illustration); 4) Diastasis is the phase of the cycle with least CMA and with least variability in longitudinal measurements; 5) Repetition is easily recognisable by trained analysts, with excellent reproducibility, but shortening and elongation occur in every OCT pullback to a greater or lesser extent, so the threshold for their identification is highly subjective, thus resulting in poorer reproducibility.

To the best of our knowledge, this is the largest study specifically dedicated to the analysis of CMA to date. Its results shed some light about the mechanism for the formation of the different subtypes of CMA. Previous studies had intuitively suggested that rotation, elongation and repetition occurred during the early ejection phase $[1,2]$, but these pioneering analyses were performed without co-registration with angiography. Indeed, some groups have suggested ECG-triggered OCT acquisition, excluding early systole, improving image quality, but disregarding potential distortion occurring in diastole [3]. The results of the current analysis, specifically focused on CMA using co-registration with angiography, demonstrate that cardiac structures appear indeed shortened during the early ejection phase. Conversely, elon- gation and repetition are solidly associated to the early rapid-inflow phase. This mechanism might be best understood with the abstract concept of relative pullback speed [3], that might be defined as (pullback + catheter speed) - tissue speed, taking as positive; the movement towards the guiding catheter and as negative; the movement fleeing away from the guiding catheter (Fig. 8). During diastasis, both the catheter and the tissue of the artery remain static, so the pullback speed is the only force to consider. At the early ejection phase, the vascular structures move towards the guiding catheter, as hypothesised by previous studies $[1,2]$, but the optical catheter is also displaced in the same direction and more intensely, because it accumulates the propelling force along the whole vessel. As a result, the relative pullback speed of the optical catheter over the vascular structures experiences a net increase, so the imaged elements appear shortened on OCT (Fig. 8). Conversely, at the early rapid-inflow phase, both the vascular structures and the optical catheter move backwards, but the latter more intensely, following the reverse reasoning, so the relative pullback speed experiences a net reduction and the imaged structures will appear elongated (Fig. 8). In extreme cases, if the relative pullback speed came to become negative, then the optical catheter would scan some vascular segments backwards and then forward again during diastole, thus creating the repetition artefact (Figs. 4, 8). Supplementary Video 1 documents the backward movement of the optical source in a paradigmatic case 


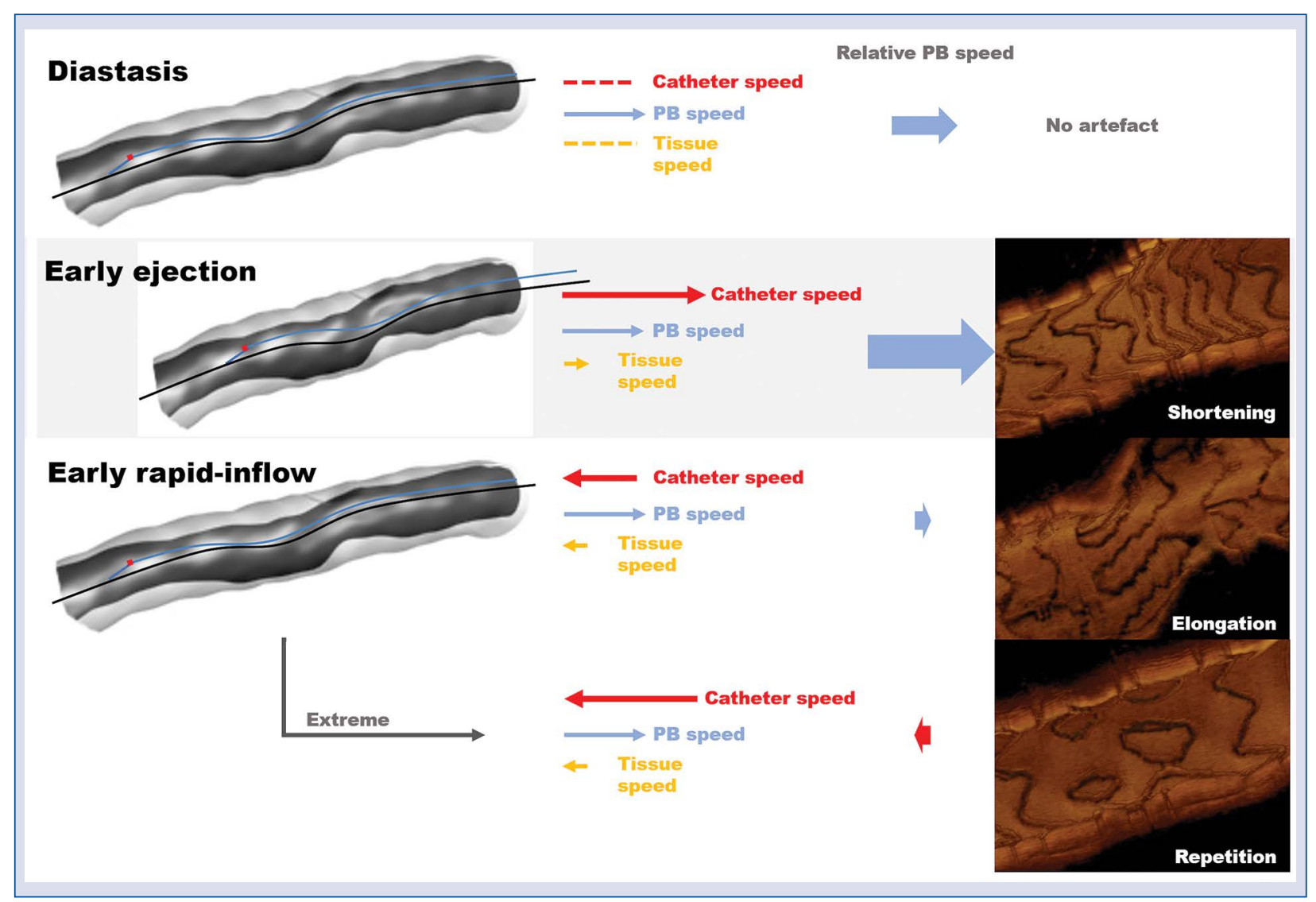

Figure 8. Mechanism of the different subtypes of cardiac motion artefact. At the early ejection phase, vascular structures and optical catheter move towards the guiding catheter, but the optical catheter moves faster, because it accumulates the propelling force along the whole vessel, resulting in increased relative pullback speed and shortening of the imaged structures. Conversely, at the early rapid-inflow phase, both the vascular structures and the optical catheter move backward, but the latter moves faster, following the reverse reasoning. The relative pullback speed is hence reduced, resulting in elongation of the imaged structures. In extreme cases, if the relative pullback speed became negative, backwards optical scanning would occur, thus creating the repetition artefact; PB — pullback; relative $\mathrm{PB}$ speed $=(\mathrm{PB}+$ catheter speed $)-$ tissue speed.

of repetition artefact, using zoomed co-registration with angiography.

Rotation is often described in both systole and diastole within the same pullback and also has poor reproducibility. This could be explained because rotation artefact is very often coupled with a sudden change in the direction of the vessel centreline during systole, subsequently corrected to the original direction during diastole, so stent structures are not properly rotated, but they simply follow the changing orientation of the vessel. The examples of rotation provided by previous studies $[1,2]$ and Figure 2 exemplify this phenomenon, that might be caused by an eccentric catheter that suddenly changes its position in the lumen cross-section during the early-ejection phase and recovers its original position during the early rapid-inflow phase, thus creating a typical C-shaped pseudocurvature in the longitudinal view of OCT, with apparent rotation at the beginning of both systole and diastole (Fig. 2) [1, 2]. This introduces some ambiguity in the definition of rotation used in the current analysis and in previous studies, this might partially explain the poor reproducibility. The present sample, although large, is insufficient to properly verify this observation, as only 6 cases of rotation were described. A specific analysis would be required in the future.

The poor reproducibility of the qualitative assessment of all subtypes of CMA, except repetition, advises against its appraisal as part of OCT studies and therefore against its request during the peer-review process, as the results would be highly variable and unlikely to add meaningful informa- 
tion. Conversely, quantitative measurements show excellent reproducibility irrespective of the phase of the cardiac cycle.

The presence of shortening and elongation to a greater or lesser extent in every OCT pullback points out the potential inaccuracy of all OCT parameters involving longitudinal measurements, like stent volume, neointimal volume [8, 9], volume of incomplete stent apposition $[9,10]$ or any measurement of length, as the same structure measured at the early rapid-inflow phase would be slightly longer than that measured during the early ejection phase. Likewise, CMA might interfere with the evaluation of longitudinal stress in vivo. The magnitude and relevance of this potential source of inaccuracy must be defined in future studies. Nonetheless, the minimal variability and excellent reproducibility of quantitative measurements during diastasis make an eventual automatic correction of this bias technically feasible in future software developments.

\section{Limitations of the study}

This is a retrospective offline analysis performed on standard real-world OCT acquisitions by trained analysts. However, the possibility of selection bias and all the intrinsic limitations to retrospective designs cannot be completely ruled out.

This study was performed with angiographic co-registration, as a best approximation to the cardiac cycle currently available. Co-registration with ECG or with the pressure waves in the polygraph would enable a more refined and accurate delimitation of the phases, in particular the atrial contraction, often elusive to detect in angiography and therefore excluded from the current analysis. Atrial contraction was encompassed at the end of the diastasis period in the current analysis, and disregarded in both the qualitative and quantitative studies. More refined studies on the topic might be performed in the future if ECG or polygraph co-registration were available.

\section{Conclusions}

Cardiac motion artefact occurs in up to $23.4 \%$ of imaged stents, but shortening of vascular structures during the early ejection phase of systole and elongation-repetition during the early rapid-inflow phase of diastole occur to a greater or lesser extent in all cases. Diastasis is free of CMAs and hence the period in which longitudinal measurements can be more consistently quantified.

Conflict of interest: None declared

\section{References}

1. Okamura T, Onuma Y, Garcia-Garcia HM, et al. High-speed intracoronary optical frequency domain imaging: implications for three-dimensional reconstruction and quantitative analysis. EuroIntervention. 2012; 7(10): 1216-1226, doi: 10.4244/EIJV7I10A194, indexed in Pubmed: 22334321.

2. Farooq V, Gogas BD, Okamura T, et al. Three-dimensional optical frequency domain imaging in conventional percutaneous coronary intervention: the potential for clinical application. Eur Heart J. 2013; 34(12): 875-885, doi: 10.1093/eurheartj/ehr409, indexed in Pubmed: 22108834.

3. Cecchetti L, Wang T, Hoogendoorn A, et al. In-vitro and in-vivo imaging of coronary artery stents with Heartbeat OCT. Int J Cardiovasc Imaging. 2020; 36(6): 1021-1029, doi: 10.1007/s10554020-01796-7, indexed in Pubmed: 32112229.

4. Prati F, Cera M, Ramazzotti V, et al. Safety and feasibility of a new non-occlusive technique for facilitated intracoronary optical coherence tomography (OCT) acquisition in various clinical and anatomical scenarios. EuroIntervention. 2007; 3(3): 365-370, doi: 10.4244/eijv3i3a66, indexed in Pubmed: 19737719.

5. Gutiérrez-Chico JL, Cortés C, Schincariol M, et al. A formula to calculate the contrast volume required for optimal imaging quality in optical coherence tomography with non-occlusive technique. Cardiol J. 2018; 25(5): 574-581, doi: 10.5603/ CJ.a2018.0112, indexed in Pubmed: 30246237.

6. Tu S, Westra J, Yang J, et al. FAVOR Pilot Trial Study Group. Diagnostic accuracy of fast computational approaches to derive fractional flow reserve from diagnostic coronary angiography: the international multicenter FAVOR pilot study. JACC Cardiovasc Interv. 2016; 9(19): 2024-2035, doi: 10.1016/j.jcin.2016.07.013, indexed in Pubmed: 27712739.

7. Cortes C, Chu M, Schincariol M, et al. Identification of the type of stent with three-dimensional optical coherence tomography: the SPQR study. EuroIntervention. 2021; 17(2): e140-e148, doi: 10.4244/EIJ-D-20-00598, indexed in Pubmed: 32928714.

8. Guagliumi G, Costa MA, Sirbu V, et al. Strut coverage and late malapposition with paclitaxel-eluting stents compared with bare metal stents in acute myocardial infarction: optical coherence tomography substudy of the Harmonizing Outcomes with Revascularization and Stents in Acute Myocardial Infarction (HORIZONS-AMI) Trial. Circulation. 2011; 123(3): 274-281, doi: 10.1161/CIRCULATIONAHA.110.963181, indexed in Pubmed: 21220730.

9. Gutiérrez-Chico JL, van Geuns RJ, Koch KT, et al. Paclitaxelcoated balloon in combination with bare metal stent for treatment of de novo coronary lesions: an optical coherence tomography first-in-human randomised trial, balloon first vs. stent first. EuroIntervention. 2011; 7(6): 711-722, doi: 10.4244/EIJV7I6A114, indexed in Pubmed: 21986329.

10. Gutiérrez-Chico JL, Wykrzykowska J, Nüesch E, et al. Vascular tissue reaction to acute malapposition in human coronary arteries: sequential assessment with optical coherence tomography. Circ Cardiovasc Interv. 2012; 5(1): 20-9, S1, doi: 10.1161/CIRCINTERVENTIONS.111.965301, indexed in Pubmed: 22319063. 